\title{
Gestion des incidents en classe, pratiques professionnelles des enseignants débutants et analyse de la plateforme nationale de ressources numériques Néopass@ction
}

\author{
Cécile Carra $^{1 *}$, Clémence Boxberger ${ }^{1}$, Bruno Robbes $^{2}$, Sébastien Pesce ${ }^{3}$ \\ ${ }^{1}$ Université d'Artois. \\ ${ }^{2}$ Université de Cergy-Pontoise. \\ ${ }^{3}$ Université François Rabelais de Tours.
}

(Received March 25, 2015; Accepted July 15, 2015)

RÉSUMÉ: Les incidents perturbent le quotidien scolaire (Barrère, 2002). Ils constituent autant d'épreuves pour les enseignants débutants. L'analyse des incidents, réalisée selon une sociologie des acteurs, donne à voir la classe comme un lieu conflictuel engageant les jeunes enseignants dans des rapports de force inter-individuels par le déploiement de "stratégies de survie » (Woods, 1977). Le sentiment d'impuissance exprimé par les jeunes enseignants face aux situations difficiles montre l'importance de la constitution de ressources de formation. Nous analyserons celles qui ont été construites dans le cadre de Néopass@ction (dirigé par L. Ria), l'intérêt de ces ressources numériques, mais aussi leurs limites.

Mots clés: Incidents en classe, épreuves enseignantes, stratégies de survie, ressources numériques, plateforme nationale Néopass@ction.

School incidents management and beginning teachers 'professional practices: an analysis of Néopass@ction, a French web-based training support

\begin{abstract}
Incidents disturb schools' everyday functioning (Barrère, 2002) and are experienced as ordeals by beginning teachers. When these incidents are analysed from a sociology of actors standpoint, the classroom appear as a polemical space; teachers engage in interpersonal power struggles by developing survival strategies (Woods, 1977). The sense of helplessness expressed by beginning teachers who are coping with critical situations show how important it is to develop training resources. Our aim is to analyse this kind of resources, namely the ones produced in the frame of the Néopass@ction project implemented in France under Luc Ria's leadership. We will insist on the relevance, but also on the limits, of such resources.
\end{abstract}

Keywords: Incidents in the classroom, teachers' ordeals, survival strategies, digital ressources, national web platform Neopass@ction. 


\section{INTRODUCTION}

Cet article se propose de déterminer les incidents typiques rencontrés par les enseignants débutants en classe, les réponses typiques qu'ils déploient (parties 1 et 2) et d'accéder au vécu professionnel en faisant émerger leurs préoccupations et émotions typiques face aux incidents (partie 3). Cette typification est à la base de la constitution des ressources numériques pour la plateforme Néopass@ction, gérée par l'IFÉ (Institut Français de l'Éducation) qui propose des ressources sur des thématiques centrales pour les enseignants : " Faire face aux incidents », « Aider les élèves », « L'entrée en classe et la mise au travail »... Ces ressources sont réalisées à partir de travaux de recherche fondés sur l'observation du travail réel des professeurs. Nous questionnerons dès lors l'intérêt et les limites de ce type de dispositif (partie 4).

En cohérence avec l'approche théorique qui sous-tend cette plateforme, et dans le but de créer des ressources sur la thématique des incidents ${ }^{1}$, notre investigation a porté sur l'activité réelle des enseignants débutants, émaillée d'incidents « ordinaires ». Ceux-ci, bien que mineurs, perturbent le quotidien scolaire (Barrère, 2002). Ils sont au cœur même du sentiment de violence des professeurs. Les enquêtes de victimation réalisées dans les pays occidentaux ont ainsi montré la rareté des faits graves, et la fréquences des micro-violences qui touchent l'école (Debarbieux, 1996 ; Gottfredson, 2001) ; ces micro-violences prenant majoritairement la forme de comportements d'indiscipline, de manquement au travail demandé et - dans une forme plus éprouvante - de conflits se construisant sur les contestations d'élèves ou de parents d'élèves (Carra, 2009 ; Carra \& Fagianelli, 2011).

Si les incidents sont de plus en plus dénoncés comme de la violence, c'est qu'ils constituent, pour les enseignants, des épreuves, traversant les sujets et renvoyant aux défis structurels qu'ils doivent affronter (Périer, 2010). Dans les écoles « difficiles » où exercent les enseignants débutants interrogés dans cet article, les relations maître-élèves sont marquées par «une interdépendance conflictuelle structurelle. Interdépendance structurelle car le décrochage de l'élève n'est pas individuel mais est une réalité collective susceptible de provoquer un risque de « décrochage du professeur », mesurable par la probabilité de burn-out, dépression, demandes de mutation et démissions. Interdépendance conflictuelle car dans ces contextes scolaires spécifiques, les buts poursuivis par les professeurs d'une part, les élèves d'autre part, sont trop discordants pour ne pas déboucher sur un conflit ouvert ou latent sur la façon dont doit se dérouler une heure de cours » (Merle, 2012, p. 141).

Faire face au désordre scolaire constitue donc une préoccupation centrale des enseignants, en particulier des débutants (Gasparini, 2013 ; Ria, 2011 ; Périer, 2010 ; Rayou et van Zanten, 2004). Elle est aussi une question de recherche ancienne (voir Waller, 1932) : la classe y apparaît alors comme un lieu conflictuel, opposant culture scolaire et culture juvénile. Si cette question a ensuite été traditionnellement étudiée sous l'angle de la discipline, l'impact des incidents sur le 
vécu professionnel conduit désormais à réinterroger les situations dans lesquelles ils apparaissent, les interactions entre enseignants et élèves et leurs effets.

\section{APPROCHE THÉORICO-MÉTHODOLOGIQUE}

Pour analyser les situations vécues comme problématiques par les enseignants, nous étudierons l'activité réelle selon une sociologie des acteurs. Contrairement à d'autres pays comme le Canada (Tardif et Lessard, 1999), l'étude du travail ordinaire au sein des classes est relativement récente en France, et plus encore lorsqu'il s'agit d'y intégrer une analyse des incidents (Barrère, 2002 ; Lantheaume et Hélou, 2008 ; Robbes, 2010). L'entrée dominante pour étudier les pratiques s'inscrit dans une perspective de professionnalisation, cette entrée constituant une des explications à cet état de fait ; la difficulté pour entrer dans les classes et réaliser de l'observation in situ en constitue une autre importante.

C'est ce défi qu'il a fallu relever pour obtenir un matériau constitué de près de 350 heures de rushes, tournés dans 14 établissements scolaires (primaire et secondaire) en 2011-2012 et 2012-2013 dans la région parisienne et dans le Nord de la France. Nous nous référerons ici à dix incidents typiques et aux entretiens d'auto-confrontation qui les accompagnent. Nous avons traité le matériau selon une double approche : une analyse thématique d'une part et une analyse de contenu d'autre part, menée à partir du logiciel Modalisa.

Ce matériau a été traité de manière à faire émerger les logiques d'acteurs, en présentant tout particulièrement dans cet article, celles des enseignants pour mettre à jour les stratégies dominantes. Celles-ci sont définies comme des actions pour « faire face » et qui se construisent en situation, par processus d'ajustement aux autres et aux objectifs ou finalités, ajustements que l'observation des situations de classe permet, seule, de repérer. L'analyse de contenu a permis d'identifier les termes les plus utilisés et les registres dans lesquels ils s'inscrivent pour caractériser les actions, réactions, préoccupations et émotions typiques des enseignants en situation.

Le sentiment d'impuissance exprimé par les jeunes enseignants face aux situations difficiles montre l'importance de la constitution de ressources visant à les accompagner dans leur professionnalisation. L'enjeu est alors de penser professionnellement les difficultés rencontrées. La plateforme nationale de ressources numériques Néopass@ction peut-elle y contribuer? Une analyse permettra d'en mettre en lumière l'intérêt mais également les possibles limites, en convoquant d'autres éléments constitutifs de la complexité des situations d'enseignement afin d'en approfondir la compréhension. Cette analyse s'appuie sur une sélection de situations. Un premier tri s'est opéré en utilisant une grille constituée d'une liste de situations-types (sur la base des cadres de référence théoriques des différents chercheurs impliqués), de l'ampleur apparente - ou commentée par les acteurs - de ces situations, du type d'objets de la conflictualisation, des acteurs impliqués. L'analyse porte à la fois sur les situations filmées et sur les entretiens d'auto-confrontation simple (Theureau, 1992) et croisés (Clot et al., 2001) tour- 
nés auprès des enseignants, de leurs collègues et de chercheurs. Ces données (le point de vue des acteurs et des experts sur la situation) ont permis d'affiner la catégorisation pour parvenir à 25 situations organisées au sein de 8 catégories. Une analyse de contenu porte à la fois sur les situations filmées dans la classe et sur les discours des acteurs, élèves compris.

\section{RÉSULTATS}

\section{Un ordre scolaire émaillé par les incidents}

\subsection{Une cristallisation des incidents qui repose sur le comportement des élèves...}

Les situations problématiques vécues par les enseignants débutants prennent typiquement la forme d'incidents, particulièrement dans « l'école de la périphérie » (van Zanten, 2001). Leur analyse à partir de situations filmées donne à voir la classe comme un lieu de désaccords multiples et récurrents. Ils se cristallisent, dans la moitié des incidents typiques retenus, sur le bruit en classe, l'agitation et les déplacements intempestifs (du bavardage au chahut anomique caractérisé (Testanière, 1967), de la remarque enjouée à l'adresse de l'enseignant à la contestation insolente, du jeu entre élèves au conflit déclaré).

Ils constituent autant de micro-ruptures à l'ordre scolaire et poussent les enseignants débutants à réagir, en manifestant leur désaccord par l'emploi de formules impératives. Le verbe « arrêter » sous sa forme impérative a ainsi été retrouvé dans l'ensemble des situations filmées, à une exception. Dans plus de la moitié des situations filmées, c'est davantage l'accumulation de micro-déviances qui fonde l'incident. De même, les entretiens font apparaître que pour près de deux tiers des enseignants débutants, c'est bien le caractère répétitif de « microindisciplines » qui constitue une préoccupation majeure : "Je dois toujours régler des conflits... ça s'insulte à longueur de journée, ça s'envoie des bouts de gomme, des capuchons... C'est constamment des conflits ! " (classe de $6^{\text {ème }}-5^{\text {ème }}$ SEGPA). L'accumulation des faits d'indiscipline, même mineurs (bavardages, manque d'attention et d'intérêt pour le travail) pose alors problème, le maintien de l'ordre constituant une préoccupation typique chez neuf enseignants sur dix.

\subsection{Et sur l'opposition au travail}

Ce souci du maintien de la discipline, déjà souligné par Gasparini (2013) ou par Rayou et van Zanten (2004), semble cacher une seconde préoccupation plus centrale : celle du travail des élèves. Les injonctions au travail sont significatives à cet égard : «Ici c'est pour travailler... Je sais, ça te fait bizarre mais c'est pour TRAVAILLER » (Classe de CM2). Ce qui fait problème pour huit enseignants sur dix, c'est avant tout le fait que certains élèves ne font pas la tâche scolaire demandée, refusent de la faire, sont en retard par rapport aux autres.... Faire ce qui est prévu, mais plus encore, exiger le travail pour tous les élèves constitue une préoccupation typique des enseignants débutants. Ces injonctions au travail don- 
nent lieu à une série d'oppositions et de contestations, se déployant en cascade et participant à la cristallisation de l'incident. Le « non » est en effet apparu de façon massive dans toutes les situations et le « mais » dans les deux tiers. Utilisés par les enseignants comme par les élèves, ces deux mots éclairent l'opposition entre des protagonistes ne partageant pas la même définition de la situation.

\subsection{Une régulation de l'incident débordant le cadre institutionnel}

Les vidéos permettent d'accéder aux formes de régulations des enseignants débutants et le registre dans lequel elles s'inscrivent. L'utilisation des phrases exclamatives (présentes dans toutes les situations filmées) ainsi que l'usage des cris (présents dans deux tiers d'entre elles) traduisent une exaspération chez l'enseignant, le conduisant dans certains cas à perdre le contrôle de lui-même ; on peut l'appréhender à partir du changement de registre de langue employée : Élève : «J'ai rien fait! » Enseignante : "T'as rien fait?! Tu te fous de moi ?? Tu te fous de moi, depuis ce matin tu n'arrêtes pas! " (classe de $6^{\text {ème}}-5^{\text {ème }}$ SEGPA) ; Enseignante : "J'en ai marre, j'ai envie de rentrer chez moi là ! (classe de $3^{\text {ème }}$ ). Les « stratégies de déstabilisation » des élèves y contribuent en mettant en question l'enseignant dans son exercice de l'autorité, ou dans les tentatives de retournement de l'ordre scolaire (des élèves qui chantent dans une classe de $4^{\text {ème }}$ par exemple). L'analyse des interactions montre, au delà de cette dimension, des incidents qui prennent la forme d'un conflit interpersonnel : «Si tu continues à m'embêter... » (classe de CM2). La réaction à l'incident n'apparaît pas médiatisée institutionnellement, comme le recours au règlement aurait pu par exemple le signifier, mais s'inscrit dans un face à face qui n'a plus pour seul enjeu que de sauver la face, de part et d'autre. Le rapport de force qui en résulte, sa nature - une confrontation interpersonnelle - sont révélateurs d'un effacement institutionnel (Dubet, 2002) et d'une désocialisation des problèmes professionnels (Barrère, 2002).

\section{Imposer son autorité}

\subsection{Ordonner, punir et exclure}

Face aux incidents, la préoccupation centrale des enseignants est de parvenir à imposer leur autorité. Cet objectif passe massivement par l'instauration d'un rapport de force. Il se caractérise par la logique d'action suivante : ordonner, punir et exclure (ou menacer de punir et d'exclure), logique présente dans presque toutes les situations filmées. Dans les entretiens, huit enseignants sur dix disent recourir à la sanction ou à la menace de sanction. Si la sanction prend régulièrement la forme de l'exclusion, c'est que cette dernière apparait comme ultime réponse pour restaurer l'ordre en classe : "Donc là j'ai pris la décision de les exclure du cours tout simplement (...). Je ne voyais plus comment les faire taire alors que c'est ce qui, pour moi, allait résoudre la situation donc je ne voulais 
plus les entendre, je voulais qu'ils se taisent et pour cela, comme je ne voyais plus comment c'était possible, il fallait qu'ils sortent de la pièce » (classe de $\left.4^{\text {ème}}\right)$.

\subsection{Marquer son territoire}

La manière dont les corps sont engagés dans l'action est révélatrice du rapport de force engagé : le positionnement des enseignants dans l'espace-classe est en effet presque systématiquement face à la source de l'incident : la classe, s'il s'agit d'un chahut ou l'élève, s'il s'agit d'un comportement perturbateur. Cette mise en scène corporelle inscrit d'emblée les protagonistes dans un rapport frontal, au sens propre comme figuré. Elle marque également une tentative de la part de l'enseignant de remettre les élèves « à leur place » selon une stratégie de domination visant à reconquérir son territoire pédagogique.

Ce face-à-face peut parfois conduire à un véritable corps-à-corps, comme en témoigne la situation de la jeune enseignante de $4^{\text {ème }} 3^{\text {ème }}$ SEGPA qui refuse de laisser un élève sortir parce que son travail n'a pas été recopié correctement. L'enseignante s'interpose entre la porte et l'élève et le repousse pour l'empêcher de quitter la salle. Chacun des protagonistes refusant de perdre la face, ils s'engagent dans une opposition corporelle prenant la forme d'un combat à remporter. Il s'agit là d'une forme d'incident déjà répertoriée dans la littérature sociologique par Anne Barrère (2002) : les incidents à la porte traduisent alors la difficulté actuelle de faire tenir de façon stable le cadre de la classe. Au delà de ce conflit de territoire, c'est bien la mise en péril de la forme scolaire dont il est question dans ce type d'incident. Cette stratégie de domination conduit à soumettre l'élève à tout prix à sa décision, au risque d'être décrédibilisé si la décision n'est pas suivie de l'effet escompté : "J'ai pris une décision et puis on va enfin y arriver et je suis aussi soulagée d'avoir pu aller jusqu'au bout de cette décision de ne pas être revenue en arrière parce que ça m'est arrivé très souvent de dire «tu vas être exclu» et puis finalement il reste dans la classe (...) et là je suis allée jusqu'au bout et donc je suis soulagée parce que ça va être fait, ce que j'ai décidé » (classe de $4{ }^{\text {ème }}$ ).

\section{L'épreuve de l'incident et ses conséquences}

\subsection{Désemparement et épuisement}

L'incident rompt le cours de l'action, submergeant les enseignants d'une émotion négative, qui peut aller de l'énervement à la sidération. Les enseignants se montrent surpris, blessés et parfois choqués par les attitudes des élèves. Ce sentiment se construit moins sur la gravité de l'incident que sur les conséquences qu'il entraîne : l'impossibilité de mener un cours, qui a pu être préparé longuement : "Pour préparer mes cours j’ai passé des heures et des heures à essayer des choses qui les intéresseraient eux, à leur âge et de travailler de manière 
un petit peu ludique (...) et finalement ça n'a pas réussi " (classe de $6^{\text {ème }}-5^{\text {ème }}$ SEGPA). Le stress naît du sentiment de perte de contrôle de la situation, de la crainte de voir l'incident se propager à l'ensemble de la classe, et au delà, de la peur de ne pas savoir faire preuve d'autorité. Il exige une réponse immédiate, explicite, non pas seulement en direction de l'élève impliqué, mais aussi en direction de la classe.

Les jeunes enseignants éprouvent in situ que leur statut et leur savoir ne leur donnent pas d'emblée une autorité sui generis, ce qui les laisse fréquemment dans un état de désemparement et d'épuisement, évoqué par la moitié des enseignants : "Dans cette classe là je n'ai pas la possibilité de les écouter et puis je ne peux plus non plus, parce qu'ils m'ont tellement épuisée... J'ai voulu être à leur écoute, j'ai essayé de l'être, mais à ce jour ce n'est plus possible. Finalement je me suis faite un peu manger par eux en essayant de les écouter donc là c'est fini... » (classe de $6^{\text {ème }} 5^{\text {ème }}$ SEGPA). Les stratégies de survie (Woods, 1977) dans lesquelles ils s'inscrivent contribuent à cet épuisement, dans le maintien d'un rapport de force quasi permanent. Les jeunes enseignants vivent ainsi des tensions, exacerbées par la confrontation à des exigences contradictoires : gérer les perturbations, canaliser les élèves difficiles, intéresser la classe, réaliser le cours prévu dans le temps imparti, couvrir le programme, le tout en tentant de gérer ses émotions.

\subsection{Engagement de soi et sentiment d'échec}

La préoccupation qu'ont les enseignants de l'image qu'ils renvoient aux élèves, ainsi d'ailleurs qu'aux parents d'élèves et aux collègues, est une des dimensions des métiers reposant sur les relations de service ; elle contribue, par l'engagement de soi qu'elle implique, à la fatigue des professionnels : « L'usure vient aussi des heures de cours qui donnent toujours lieu à un exercice de présentation de soi et de justification de son action dans l'interaction avec des groupes à l'esprit critique aiguisé et peu prêts à s'en laisser conter. L'engagement de soi demandé pour faire tenir les situations conduit à un état de fatigue très prononcé. » (Lantheaume \& Hélou, 2008, p. 66).

Les réponses apportées le plus souvent, telles les punitions, menaces et exclusions sont jugées inefficaces par les enseignants eux-mêmes. Certains les perçoivent comme signe d'échec, voire de renoncement à certains idéaux : "Pour moi les exclure c'est finalement abandonner, c'est dire je n'y arrive plus donc je les confie à quelqu'un d'autre. Mais étant donné qu'ils en étaient à se battre en classe, de toute façon ce n'est pas une situation où l'on peut travailler avec les élèves, et du coup à ce moment-là j'ai préféré les sortir 》 (classe de $6^{\text {ème }}-5^{\text {ème }}$ SEGPA). S'ensuit, chez huit enseignants sur dix, un sentiment d'impuissance voire de résignation : "Mais là je me sens quand même impuissante parce que ça va vraiment dans tous les sens » (classe de $3^{\text {ème}}$ ). Pour autant, plus de la moitié des enseignants disent ne pas connaître d'alternative : "Moi je ne sais pas ce qu'il faudrait mettre en place pour dissuader ces élèves » (classe de CM2). Le 
sentiment d'échec, du fait d'un engagement de soi important, est alors d'autant plus lourd à porter.

\subsection{Processus d'ajustement et protection de soi}

La réalité de l'exercice du métier conduit alors à des processus d'ajustement ayant parfois pour conséquence la détérioration de l'image de soi : "Là je peux vraiment m'énerver.. Mais après je sais que souvent quand je rentre chez moi je me dis " pourquoi tu as crié autant? " (classe de CM1). Ces processus peuvent conduire à une baisse des exigences de travail, qui s'exprime par la résignation face à des situations que l'enseignant ne peut faire évoluer : "Mais je ne sais pas comment j'aurai pu faire, de toute manière je ne peux plus rien en tirer donc... » (classe de $4^{\text {ème }} 3^{\text {ème }}$ SEGPA). Parfois c'est le principe même d'éducabilité qui est abandonné, l'échec étant renvoyé à des causes externes : c'est tantôt le manque de volonté des élèves qui est pointé : «Je me pose la question que faire pour faire travailler les élèves qui eux-mêmes ne veulent pas travailler ou ne sont pas en capacité de travailler... " (classe de seconde) ; tantôt les déficiences éducatives du milieu des élèves : "S'il est comme ça ce n'est pas de ma faute, je ne suis pas responsable du fait qu'il soit troublé, qu'il ait des problèmes d'éducation... Moi en tant qu'enseignante je ne peux pas gérer ça » (classe de CM2).

Enfin, les processus d'ajustement des enseignants peuvent consister en un désengagement visant à se préserver. Cette protection de soi peut passer par la décision d'une exclusion ou d'une punition collective permettant de gérer la charge émotionnelle du moment : "Je sais qu'à ce moment là ce n'est pas la meilleure solution de leur donner une punition collective mais c'est simplement pour avoir une minute pour moi, pour ne plus les voir et respirer un petit peu (...) Mais rien qu'avoir une minute où je ne les vois plus, où je leur tourne le dos, ça m'a déjà permis de me calmer un peu à ce moment là. Là, c'est pour moi, pour me préserver à ce moment là. Là, je ne pense pas à eux mais à moi à ce moment là " (classe de $6^{\text {ème }}-5^{\text {ème }}$ SEGPA).

Dans un contexte où l'ordre scolaire semble devoir être sans cesse reconstruit (Prairat, 2012), les enseignants se voient contraints à des formes d'engagement de soi très fortes, d'autant plus fortes que la profession ne semble pas offrir de supports sociaux à l'activité dans un contexte de désocialisation des problèmes professionnels (Barrère, 2002). Ce qui les conduit à un travail de recherche de solutions, épuisant tant sur le plan physique que moral quand il est réalisé dans la solitude.

\section{Analyse des ressources en lignenéopass@ction}

Comment dès lors, aider les enseignants débutants à passer de «stratégies de survie » à la construction de savoirs d'action (Argyris \& Schön, 1974 ; Schön, 1983 ) et de gestes professionnels (Bucheton, 2009) qui leur permettraient de développer leur professionnalité, dans les « deux fonctions principales » de 
l'activité d'enseignement : « conduire la classe (...) et instruire les élèves » (Durand, 1996, p. 109) ? La plateforme Néopass@ction veut apporter des réponses à cette question, en mettant à la disposition des enseignants novices et de leurs formateurs des vidéos présentant des situations d'enseignement correspondant à des préoccupations de début de carrière. Ces situations sont d'abord montrées, puis commentées ou analysées par des professeurs novices et expérimentés, enfin par des chercheurs.

\subsection{L'activité effective comme socle de formation}

La démarche de formation, basée sur l'analyse de l'activité professionnelle ${ }^{2}$ effective des enseignants débutants en situation, est sous-tendue par plusieurs référents théoriques : l'ergonomie de langue française qui distingue tâche et activité, activité prescrite et activité effectivement réalisée, activité réalisée et activité réelle (Ria, 2008); la clinique de l'activité (Clot, 1999) qui intègre l'ergonomie, la clinique, la psychologie à l'activité et « explore les conditions du développement du pouvoir d'agir des acteurs dans et par le travail » (Danvers, 2010, p. 111) ; l'entretien d'explicitation (Vermersch, 1994), qui vise à mettre au jour le fonctionnement cognitif préréfléchi de l'acteur (routines, savoirs non conscients), en l'aidant à verbaliser le déroulement de ses actions; le « cours d'action» (Theureau, 2004), qui s'intéresse aux dynamiques de construction du sens par l'acteur grâce à l'entretien d'autoconfrontation ; l'entretien d'autoconfrontation croisé (Clot, Faïta, Fernandez \& Scheller, 2001), qui permet à deux enseignants de confronter leurs styles individuels comparativement au genre en usage dans la profession ; la didactique professionnelle (Pastré, 2005), qui, à partir de situations scénarisées typiques, confronte « les acteurs à une complexité proche des situations réelles tout en favorisant la construction/déconstruction «d'une expérience sans risque $(\ldots)$ » notamment en recourant à la manipulation des paramètres de la situation » (Ria, 2008, p. 284). Il s'agit ainsi de « constituer un observatoire de situations professionnelles réelles » (ibid.). L'approche épistémologique est énactive, soutenant " l'idée qu'à chaque instant, l'acteur «fait émerger» le monde de son action en relation avec son engagement dans l'environnement et que l'action et la cognition sont inséparables » (Leblanc, Ria, Dieumegard, Serres \& Durand, 2008, p. 62).

\subsection{Un dispositif contribuant à la professionnalisation}

L'approche de la formation développée par Néopass@ction va à l'encontre de modèles qui reposent principalement sur les logiques prescriptives expertes, privilégient les contenus de savoirs disciplinaires, séparent la conduite de classe des fonctions didactique et pédagogique (en situation d'opposition au travail par exemple, les savoirs d'action qui concernent la présentation des savoirs, la clarté des consignes sont considérés comme la variété des situations d'apprentissage ou l'explicitation par l'enseignant de ce qui n'est pas discutable). Ainsi, elle rejette 
les apports généraux et abstraits, qu'il est difficile de traduire en actes pertinents. Elle exclut également les apports portant sur des méthodes, trop éloignées des compétences des professeurs novices. Ses atouts résident dans le fait qu'elle s'appuie prioritairement sur les situations vécues par les débutants (mobilisant secondairement l'activité d'enseignants plus expérimentés) pour explorer des enjeux liés à l'activité. La démarche associe aux vidéos de classe, la mise en mots des vécus professionnels pour en comprendre le sens, l'efficience, les aspects spécifiques et typiques, les ancrages concrets dans l'action, les contraintes liées aux conditions d'enseignement, etc. Elle recense, décrit, analyse les diverses modalités d'enseignement et en interroge la pertinence, l'efficacité attendue, supposée... Elle propose pour chaque situation le regard croisé d'enseignants débutants, expérimentés et de chercheurs, montrant ainsi la complexité du travail enseignant et sa dynamique de transformation ${ }^{3}$. Ainsi, elle permet aux enseignants novices de se reconnaître dans l'activité de leurs pairs, de se déculpabiliser lorsqu'ils rencontrent des situations difficiles (on s'interdit tout jugement des personnes, comme dans les situations où des enseignants mettent des punitions collectives ou excluent de cours), en identifiant les caractéristiques typiques de l'activité débutante et ses effets sur les élèves, de comprendre la dynamique de transformation de cette activité (et de la leur), d'accéder à d'autres modalités d'actions possibles (savoirs d'action et gestes professionnels utilisables dans leurs classes pour dépasser les situations critiques, comme différer vers un lieu de parole dédié le règlement d'un conflit, adapter son positionnement dans l'espace ou régler sa proximité/distance à l'élève, annoncer des décisions que l'on peut tenir), d'anticiper des situations non rencontrées. Le travail enseignant y est exposé dans sa complexité, avec ses dilemmes récurrents constitutifs et ses compromis fragiles. Un tel dispositif est professionnalisant au sens où il supporte les transformations du travail enseignant, contrairement aux réponses de type « recettes » qui refusent ces réalités. La formation s'inscrit dans une temporalité longue, qui implique les acteurs (interactivité).

\subsection{Limites de cette ressource pour la formation}

Le modèle de formation sur lequel s'appuie la plateforme Néopass@ction peut cependant être discuté. Premièrement, la centration sur le geste enseignant évacue d'autres aspects constitutifs d'une situation. À l'évidence, la sélection d'une partie des données produit un effet de cadrage : on étudie l'enseignant dans sa classe, sur des séquences temporelles courtes, dont la sélection par les chercheurs implique une définition préalable de la situation : c'est ainsi généralement le concepteur et non l'enseignant filmé qui choisit quelques moments de la situation ou difficultés sur lesquels les analyses se focalisent, parce qu'il les considère a priori comme typiques de cette situation. D'une part, cette approche réinjecte des catégories exogènes. D'autre part, elle privilégie des gestes ponctuels (plutôt notamment que des situations complexes) comme porteurs de significations. Un risque de morcellement, voire de simplification de l'activité est à redouter lors- 
que la démarche oriente de fait les préoccupations des enseignants. La formation pourrait alors conduire à des recommandations comportementales « hors sol ", qui prendraient pour seul critère le geste efficace ou les pratiques expertes.

Deuxièmement, si l'on considère que la pratique enseignante ne se réduit pas à des gestes, que l'on ne comprend véritablement une situation professionnelle qu'en étudiant des interactions en contexte, des significations sont aussi à chercher dans d'autres éléments constitutifs de la situation. Il y a tout d'abord des éléments de contexte sociologiques, économiques, culturels et institutionnels qui déterminent en partie les situations scolaires. Dans la classe, citons les dimensions pédagogiques ${ }^{4}$ et didactiques ${ }^{5}$ de son fonctionnement (avec la part d'idéal qu'elles comportent, nécessaire pour entretenir le désir de l'enseignant), le sens des situations d'enseignement/apprentissage et les rapports aux savoirs à acquérir, les aspects groupaux, ceux relevant d'un travail en équipe pédagogique ; autant d'éléments qui peuvent être oubliés dans la pratique et l'analyse réflexive, renforçant certaines formes scolaires. Indiquons encore le risque d'évacuer les significations inconscientes (au sens psychanalytique) des situations et des gestes, et plus généralement les dimensions complexes et situées des processus de construction du sens de l'expérience (Pesce, 2015). On risque alors de suggérer que toute action serait toujours rationnelle alors qu'il n'en est rien : pensons par exemple à ces actes-réflexes posés sous le coup de l'émotion, à ces « ratés » qui révèlent, à leur insu, la part d'inconscient des sujets.

Enfin, l'élève n'est-il pas le point aveugle du modèle de formation ? L'enseignant ne dispose d'aucun moyen d'accéder à sa « lecture » de la situation (définition, compréhension, analyse, interprétation, sens, investissement symbolique), à ses intentions lorsqu'il agit en réponse à ses demandes, car les paroles de l'élève ne sont pas analysées. Or, nous pensons qu'il est possible d'aller plus loin dans la compréhension de certaines situations - notamment celles de chahuts supposés « anomiques » (Testanière, 1967) -, en dépassant l'absence apparente de règles pour les considérer comme porteuses de significations objectivables et de caractéristiques identifiables. Pour cela, l'accès à la façon dont l'élève décrypte la situation, en interaction avec son professeur et la classe, est indispensable.

\section{DISCUSSION ET CONCLUSIONS}

Après avoir déterminé les incidents typiques rencontrés par les enseignants débutants, nous nous sommes engagés à identifier les logiques d'actions des professeurs en les caractérisant à trois niveaux : les réponses dominantes employées pour faire face aux incidents, les préoccupations et les émotions qui sous-tendaient de telles situations.

Les données recueillies montrent un incident typique prenant la forme de micro-déviances d'élèves perturbant l'ordre scolaire. Si un recueil de données par entretien ou par questionnaire permettrait difficilement de saisir l'impact de tels faits sur le vécu professionnel, l'observation in situ contribue en revanche à ancrer l'analyse sur des situations scolaires réelles et à accéder aux logiques 
d'acteurs - élèves et enseignants - qui infléchissent le cours d'action. Les entretiens avec les enseignants, sur les situations problématiques qu'ils rencontrent, permettent d'appréhender leur lecture de la réalité et de comprendre leurs réponses.

Une double préoccupation typique apparaît ainsi au cœur des logiques d'action des jeunes professeurs : le travail et la discipline. L'incident leur apparaît comme une menace, mettant en péril le travail de la classe mais aussi leur autorité. L'urgence est alors de reprendre le contrôle de la situation. Les réponses typiques relèvent massivement des sanctions et, tout particulièrement de l'exclusion. Elles les engagent dans des rapports de force inter-individuels qui les conduisent à recourir à des « stratégies de survie ». Celles-ci sont constituées des actions-réactions des professeurs à des situations dans lesquelles ils sont « pris au piège », dans l'objectif de « sauver les apparences » et d'échapper à la destruction de leur identité. Or, ce type de réponse, jugé inefficace par nombre d'enseignants, les engage dans un rapport de force épuisant avec le ou les élève(s) concerné(s).

Les incidents déstabilisent ainsi les jeunes professeurs, cette déstabilisation se manifestant par un débordement émotionnel. Les émotions naissent d'un décalage entre attentes de l'enseignant et la situation telle qu'il la perçoit. Elles traduisent simultanément un rapport aux normes et aux buts mais aussi, le potentiel (ou son manque) de maîtrise de la situation que l'acteur s'attribue. Ce débordement émotionnel, en inscrivant la régulation de l'incident dans un faceà-face, en enfermant les enseignants dans des rapports de force inter-individuels, met en péril un positionnement professionnel, exposant les professionnels à des relations à risque.

Les incidents apparaissent finalement comme une réalité dont la gestion est constitutive du métier. La socialisation des problèmes professionnels liés à l'autorité apparaît par conséquent nécessaire : la plateforme Néopass@ction constitue dès lors un dispositif spécifique contribuant au développement d'une telle professionnalité. Cette plateforme permet en effet d'engager les enseignants débutants dans une analyse réflexive et évolutive de leur activité à partir des difficultés effectives qu'ils rencontrent, dans la perspective d'accompagner leur professionnalisation. Toutefois, un dispositif de formation, aussi pertinent soit-il, ne peut constituer le tout d'une formation. Les limites que nous avons mises au jour montrent qu'une situation professionnelle ne se réduit pas à ne voir que « la partie émergée de l'iceberg $\gg 6$. L'important est d'utiliser cette plateforme pour professionnaliser les enseignants là où elle est la plus pertinente, c'est-à-dire pour développer de manière réflexive leurs savoirs d'action et gestes professionnels alternatifs.

\section{NOTES}

[1] La plateforme est accessible à l'adresse http://neo.ens-lyon.fr/neo.

[2] Des repères théoriques sont proposés sur la plateforme : http://ife.ens- 
lyon.fr/formation-formateurs/une-mission-formation-qui-se-structure/professionnalite-enseignante-1/analyse-du-travail

[3] D'après Ria, L. (2011, février). Charte de cadrage pour la construction de ressources. 5 pages, non publié.

[4] La pédagogie est un champ de recherches et de pratiques qui s'intéresse à la situation d'enseignement en portant notamment son attention sur les relations maître-élèves ou entre élèves, les aspects organisationnels, matériels et techniques en tant qu'ils participent des conditions d'apprentissage. Selon Meirieu (2005), elle mobilise et articule trois pôles : un pôle axiologique (valeurs), un pôle scientifique (connaissances scientifiques) et un pôle praxéologique (outils et instruments pour l'action).

[5] La didactique (ou plutôt les didactiques) est un champ de recherche, qui regroupe « les disciplines de recherche qui analysent les contenus (savoirs, savoir-faire...) en tant qu'ils sont objets d'enseignement et d'apprentissage, référés/référables à des matières scolaires » (Reuter, 2010, p. 69).

[6] Ce dont les concepteurs de Néopass@ction sont bien conscients : http:// neo.ens-lyon.fr/neo/formation/presupposes-theoriques/principes-ethiques-pourla-videoformation

\section{RÉFÉRENCES}

Argyris, C., et Schön, D. A. (1974). Theory in Practice. Increasing Professional Effectiveness. San Francisco : Jossey-Bass.

Barrère, A. (2002). Un nouvel âge du désordre scolaire : Les enseignants face aux incidents. Déviance et société, 26, $n^{\circ} 1,3-19$.

Bucheton, D. (dir.), (2009). L'agir enseignant : des gestes professionnels ajustés. Toulouse : Octarès.

Carra, C. (2009). Violence à l'école élémentaire : l'expérience des élèves et des enseignants. Paris : PUF.

Carra, C., et Faggianelli, D. (2011). Les violences à l'école. Paris : PUF.

Clot, Y. (1999). La fonction psychologique du travail. Paris : PUF.

Clot, Y., Faïta, D., Fernandez, G., et Scheller, L. (2001). Entretiens en a u t o confrontation croisée : une méthode en clinique de l'activité. Éducation Permanente, 146 (1), 17-25.

Danvers, F. (2010). Autour des mots de la formation. «Clinique ». Recherche \& formation, 63, 105-116.

Debarbieux, E. (1996). La violence en milieu scolaire. Paris : ESF Éditeur.

Dubet, F. (2002). Le déclin de l'institution. Paris : Seuil.

Durand, M. (1996). L'enseignement en milieu scolaire. Paris : PUF.

Gasparini, R. (2013). La discipline au collège. Une analyse sociologique de la note de vie scolaire. Paris : Presses universitaires de France.

Gottfredson, D. (2001). Schools and Delinquency. Cambridge : University Press.

Lantheaume, F., et Hélou, C. (2008). La souffrance des enseignants, une sociologie pragmatique du travail. Paris : PUF. 
Leblanc, S., Ria, L., Dieumegard, G., Serres, G., et Durand, M. (2008). Concevoir des dispositifs de formation professionnelle des enseignants à partir de l'analyse de l'activité dans une approche énactive. Activités, 5 (1), 150-172.

Meirieu, P. (2005). Méthodes pédagogiques. In Champy, P., et Étévé, C. Dictionnaire encyclopédique de l'éducation et de la formation (pp. 630-635). Paris : Retz.

Merle P. (2012). Violences à l'école : Quelques constats et perspectives de changement. In B. Galand, C. Carra, et M. Verhoeven (eds.), Prévenir les violences à l'école (pp. 137-149). Paris : PUF.

Pastré, P. (2005). Apprendre par la simulation : de l'analyse du travail aux apprentissages professionnels. Toulouse : Octarès.

Périer, P. (2010). L’ordre scolaire négocié. Parents, élèves, professeurs dans les contextes difficiles. Rennes : PUR.

Pesce, S. (2015). La « violence en institution » comme effet de sens en contexte... Vers une responsabilité assumée du sujet collectif. In R. Casanova et S. Pesce (dir.), La violence en institution. Situations critiques et significations (pp. 7-48). Rennes : PUR.

Prairat, E. (2012). Comprendre la question de la norme. In B. Galand, C. Carra, et M. Verhoeven (eds), Prévenir les violences à l'école (pp. 217-228). Paris : PUF.

Rayou, P. et Van Zanten, A. (2004). Enquête sur les nouveaux enseignants. Paris : Éditions Bayard.

Reuter, Y. (dir.). (2010). Dictionnaire des concepts fondamentaux des didactiques. Bruxelles : De Bœck.

Ria, L. (2008). Ergonomie cognitive du travail enseignant. In A. Van Zanten (dir.), Dictionnaire de l'Éducation (pp. 282-284). Paris : PUF.

Ria, L. (dir.), (2010). Plateforme de formation en ligne Néopass@ction de l'Institut Français de l'Education de l'ENS de Lyon.

Ria, L. (2011). Les stratégies et dilemmes des enseignants débutant en milieu difficile. Les cahiers pédagogiques, 488, 46-48.

Robbes, B. (2010). L'autorité éducative dans la classe. Douze situations pour apprendre à l'exercer. Paris : ESF.

Schön, D. A. (1983). The Reflective Practitioner. How Professionnals Think in Action. New York: Basic Books.

Tardif, M. et Lessard, C. (1999). Le travail enseignant au quotidien. Expérience, interactions humaines et dilemmes professionnels. Bruxelles : De Boeck Université.

Testanière, J. (1967). Chahut traditionnel et chahut anomique dans l'enseignement du second degré. Revue Française de sociologie, vol. 8, $n^{\circ}$ hors série 1, $17-$ 33.

Theureau, J. (2004). Le cours d'action : méthode élémentaire. Toulouse : Octarès.

Theureau, J. (1992). Le cours d'action : analyse sémio-logique. Essai d'une anthropologie cognitive située. Berne : Peter Lang. 
Vermersch, P. (1994). L'entretien d'explicitation en formation initiale et en formation continue. Paris : ESF.

Waller, W. (1932). The sociology of teaching. New-York : J. Wiley \& sons, inc..

Woods, P. (1977). Teaching for Survival. In P. Woods et M. Hammersley (eds). School experience - Explorations in the Sociology of Education (pp. 272293). London : Croom Helm. Texte repris dans Forquin, J.-C. (1997). Les sociologues de l'éducation américains et britanniques. Bruxelles : De Boeck Université, p. 351-376.

Zanten van, A. (2001). L'école de la périphérie. Scolarité et ségrégation en banlieue. Paris : PUF. 